\title{
Síntomas de ansiedad, depresión y factores psicosociales en hombres que solicitan atención de salud en el primer nivel
}

\author{
Ma Consuelo Espinosa Muñoz, ${ }^{1}$ Luz Adriana Orozco Ramírez, ' José Luis Ybarra Sagarduy'
}

Artículo original

\section{ABSTRACT}

\section{Background}

Anxiety and mood disorders are the cause of psychological deterioration in the world. In the case of men, specific somatic symptomatology can be present that indicate depression or anxiety but, because of their masculine socialization, they tend to suppress or minimize their psychological suffering or impose barriers that keep them from identifying their internal emotions and the symptoms of a serious mental issue.

\section{Objective}

The purpose of this was to determine whether there are significant relationships between symptoms of anxiety, depression and other psychosocial factors along with the consultation motive of the adult male population who demand first level health care.

\section{Method}

The sample considered for this study was 276 male subjects aged 18 to 65 who were attended as outpatients in various health institutions in Ciudad Victoria, Tamaulipas. With this sample, the Goldberg Scale of Anxiety and Depression and the Scale of Psychosocial Operation were used.

\section{Results}

It was found that $57 \%(n=157)$ were diagnosed with a prevalence of anxiety symptoms and $49 \%(n=135)$ were diagnosed with symptoms of depression. The results obtained confirm that among the factors that cause these symptoms are life stress, negative exchange among interactions, negative health habits and an economic income lower than 1000 pesos.

\section{Discussion and conclusion}

These results also confirm the relationship among symptoms of anxiety, depression, psychosocial factors and deteriorating health.

Key words: Anxiety, depression, men, psychosocial factors, primary care.

\section{RESUMEN}

\section{Antecedentes}

Los trastornos de ansiedad y del estado de ánimo son causa de de terioro psicológico. En el caso específico de los hombres, pueden presentar sintomatología clínica somática de depresión y ansiedad, pero, debido a la socialización masculina, pueden sentirse inclinados a ocultar o minimizar su sufrimiento psicológico o imponerse barreras que les impidan identificar y comunicar emociones internas y mucho menos a valorar estas manifestaciones como indicadores de un problema de salud mental.

\section{Objetivo}

El presente trabajo tiene como objetivo determinar las relaciones existentes entre síntomas de ansiedad y depresión, factores psicosociales y el motivo de consulta en población adulta masculina que demanda atención de salud en primer nivel.

\section{Método}

Los participantes fueron 276 sujetos masculinos de 18 a 65 años atendidos en consulta externa de diversas instituciones de salud de Ciudad Victoria, Tamaulipas. Se aplicó a esta muestra la Escala de Ansiedad y Depresión de Goldberg y la Escala de Funcionamiento Psicosocial.

\section{Resultados}

Se encontró una prevalencia para síntomas de ansiedad de $57 \%$ ( $n=157)$ y de síntomas de depresión de $49 \%$ ( $n=135)$. Los resultados mostraron que entre los factores predictores de esta sintomatología se encuentran el estrés de vida, el intercambio negativo en sus interacciones, los hábitos negativos de salud y un ingreso económico menor a 1000 pesos.

\section{Discusión y conclusión}

La presente investigación viene a confirmar una relación entre síntomas de ansiedad, depresión, factores psicosociales y el deterioro de la salud.

Palabras clave: Ansiedad, depresión, hombres, factores psicosociales, atención primaria.

Unidad Académica de Trabajo Social y Ciencias para el Desarrollo Humano. Universidad Autónoma de Tamaulipas.

Correspondencia: Mtra. Ma. Consuelo Espinosa Muñoz. Centro Universitario Adolfo López Mateos s/n, Universidad Autónoma de Tamaulipas, 87000, Cd. Victoria, Tamps., México. Tel: (834) 318-1730 Ext. 2242. E-mail: lapsicologaesconsuelo@hotmail.com

Recibido primera versión: 25 de marzo de 2013. Segunda versión: 17 de agosto de 2014. Aceptado: 5 de noviembre de 2014. 


\section{ANTECEDENTES}

En México, la Secretaría de Salud (SSA) refiere que en las instituciones públicas de salud se proporcionaron durante 2009 más de doscientos nueve mil millones de consultas externas generales, que atendieron demandas de atención prenatal y enfermedades infecciosas, así como a usuarios con patologías crónicas. ${ }^{1}$ Sin embargo, solamente $1 \%$ de la población atendida tuvo como motivo de consulta su salud mental. ${ }^{2}$

Estos datos permiten suponer que, en el primer nivel de atención los padecimientos mentales permanecen subdiagnosticados. No obstante, en México la Encuesta Nacional de Epidemiología Psiquiátrica (ENEP) reporta que $28.6 \%$ de la población mexicana presentó alguno de los 23 trastornos mentales de la Clasificación Internacional de Enfermedades (CIE), siendo los más prevalentes los trastornos de ansiedad, el abuso de sustancias y los trastornos del estado de ánimo. ${ }^{3}$

Las repercusiones individuales, familiares y sociales de estos padecimientos generan una disminución en la productividad. En la depresión, la evidencia empírica da cuenta de la discapacidad que genera, que es mayor que la de otras enfermedades crónicas como diabetes o hipertensión. ${ }^{4}$ Además, está considerada como una de las causas de años saludables de vida perdidos. ${ }^{5}$ Por otro lado, la depresión en comorbilidad con una enfermedad física exacerba el dolor e interfiere con la adherencia al tratamiento. ${ }^{6}$

Asimismo, la presencia de depresión puede afectar negativamente las relaciones interpersonales. En los padres, por ejemplo, se ha asociado a patrones disfuncionales de crianza con repercusiones en la salud mental de sus hijos. ${ }^{7,8}$

Estos padecimientos los presentan más mujeres que hombres. De acuerdo con los datos de la ENEP, se observa en las mujeres una prevalencia de $11.2 \%$ y en los hombres de $6.7 \%$ para cualquier trastorno afectivo, y en los trastornos de ansiedad la prevalencia de presentar éstos alguna vez en su vida en las mujeres fue de $18.5 \%$ y en hombres de $9.5 \%{ }^{3}$

Las causas por las que se desarrollan los trastornos mentales no son del todo bien conocidas, pero se ha reconocido que la etiología es multifactorial y así se tienen referentes para corroborar un diagnóstico de ansiedad o depresión con factores biológicos, ${ }^{9-11}$ y psicosociales. ${ }^{12-17}$

La salud mental en el género masculino tiene características que la hacen merecedora de especial atención, siendo la depresión un aspecto preocupante por la correlación con la conducta suicida. En México, las estadísticas reportan que en 2007 se observaron 4388 suicidios en ambos sexos. Como causas se identificaron la ansiedad, depresión, estrés, soledad, desesperanza, exigencia social y sentimientos de rechazo. ${ }^{18}$

Las entidades federativas con mayor prevalencia de suicidios en hombres fueron las de la zona norte (Baja California, Sonora y Chihuahua) y sur-sureste del país (Tabasco, Campeche y Quintana Roo). Si bien es cierto que son más las mujeres las que intentan suicidarse, paradójicamente son más los hombres que mueren por lesión autolítica al utilizar medios más letales e irreversibles. ${ }^{18,19}$

El perfil sintomático de la depresión en los hombres es, en general, diferente al femenino, con un predominio de la fatiga, insomnio, irritabilidad y pérdida de interés por el trabajo y el tiempo libre. En comparación con las mujeres, los hombres deprimidos expresan menos sentimientos de tristeza y son menos propensos a la baja autoestima y a los sentimientos de culpa excesiva. ${ }^{20}$

Estudios epidemiológicos ${ }^{21,22}$ relacionan un mayor abuso de alcohol y drogas en sujetos masculinos con trastornos de ansiedad y del afecto. Así, en lugar de reconocer sus síntomas, pedir ayuda o solicitar tratamiento apropiado, los varones pueden recurrir al alcohol o a las drogas cuando están deprimidos, frustrados, desanimados, enojados, irritables y algunas veces violentamente abusivos. ${ }^{23}$ Otros tienden a buscar refugio en el trabajo o a participar en comportamientos de asunción de riesgo. ${ }^{24}$ Además, hay culturas y factores sociales que pueden ejercer presión sobre los hombres para presentar una imagen de fuerza y la depresión se considera aquí como un signo de debilidad o falta de autocontrol, lo que puede conducir a sentimientos de tristeza que se canalizan en hostilidad. ${ }^{25,26}$

Estos malestares antes mencionados en los varones, se han reconocido poco y se han abordado menos. ${ }^{27}$ Las causas pueden estar en la medición, como sesgo de género presente en muchas pruebas, lo que lleva al subregistro de la ansiedad y depresión en el caso de los hombres, dadas las características de estos autoinformes que parecen evaluar rasgos típicamente femeninos, más que síntomas de ansiedad y depresión en sí mismos. ${ }^{28}$ Por otro lado, los varones acuden con menor frecuencia que las mujeres a buscar ayuda para problemas psiquiátricos, tanto en servicios especializados como en atención primaria. ${ }^{20}$ Como causas de esta conducta se identifica la forma en que los hombres perciben, valoran y comunican los síntomas, ${ }^{29}$ y otras posibles causas son, por ejemplo, el temor a perder dinero o no acudir a trabajar. ${ }^{30}$

Teniendo en cuenta lo anterior, el interés del presente trabajo se centra en analizar la relación de los síntomas de ansiedad y depresión con los factores psicosociales y el motivo de consulta en la población masculina que solicita atención de salud en primer nivel.

\section{MÉTODO}

Se trata de un estudio cuantitativo, no experimental, transversal con un alcance descriptivo-correlacional.

\section{Participantes}

Participaron en el estudio 276 sujetos masculinos de 18 a 65 años que solicitaban atención médica en distintas instituciones de salud en Ciudad Victoria, Tamaulipas, México. 
Para calcular la muestra por estudiar, se procedió a solicitar el número de pacientes masculinos atendidos el mes anterior en las instituciones de primer nivel. Con un universo de 7490 usuarios masculinos atendidos por mes, se procedió a calcular el tamaño de la muestra representativa, teniendo en cuenta un error máximo de $5 \%$, un porcentaje estimado de casos de $25 \%$, según estudios previos, ${ }^{31-33}$ y un nivel de confianza de 95\%. El tamaño resultante requerido para que la muestra sea representativa es de 277 usuarios masculinos del sector salud que acuden a los centros en demanda de atención médica en los consultorios de atención primaria. Delimitada la muestra, se procedió a calcular el número de usuarios entrevistados por institución utilizando como método el muestreo aleatorio estratificado por afijación simple, considerando como estratos los distintos centros de salud. Por último, se seleccionaban los pacientes que acudían a las distintas consultas mediante muestreo aleatorio sistemático.

El estudio fue aprobado por la comisión dictaminadora del Departamento de Enseñanza e Investigación del Hospital General Dr. Norberto Treviño Zapata, Clínica Hospital del Instituto de Seguridad y Servicios Sociales de los Trabajadores del Estado (ISSSTE) y la Jurisdicción Sanitaria $N^{\circ} 1$ en Ciudad Victoria, Tamaulipas, México.

En los datos sociodemográficos (cuadro 1) se observa un predominio de edad en el rango de 30 a 49 años $(\bar{X}=43.35$ D.E $=13.1)$, con un porcentaje del $43.1 \%(n=119)$. Coexiste en pareja $73.6 \%(n=203)$ y 93\% $(n=257)$ tiene como residencia la zona urbana. Además, $60 \%(n=164)$ de los hombres no cuentan con estudios profesionales (licenciatura), lo que se refleja en su percepción económica, pues 68\% ( $n=189)$ perciben un salario menor de cinco mil pesos. Se observa que $51.8 \%(n=143)$ viven con esposa e hijos.

\section{Instrumentos}

Se diseñó un instrumento que contenía los siguientes cuestionarios y escalas:

a) Cuestionario de datos sociodemográficos. Con el que se pregunta sobre la edad, estado civil, área de residencia, grado de escolaridad, ingreso mensual y personas con las que se vive.

b) Escala de depresión-Ansiedad de Goldberg, ${ }^{34}$ en su versión castellana, que ha demostrado fiabilidad y validez en el ámbito de la atención primaria y presenta una sensibilidad de $83.1 \%$, una especificidad de $81.8 \%$ y un valor predictivo de $95.3 \% .{ }^{35}$ Es una prueba de cribaje que consta de dos subescalas: una para la detección de síntomas de ansiedad y otra para síntomas de depresión, conformadas por nueve preguntas con respuesta dicotómica (sí/no). En la muestra de este estudio, se obtuvo un coeficiente alfa de consistencia interna de 0.85 para síntomas de ansiedad y 0.83 para síntomas de depresión. c) Escala de funcionamiento psicosocial (EFP). Esta evalúa el funcionamiento integral de la salud en población mexicana. ${ }^{36}$ Consta de 37 reactivos en formato tipo Likert con cinco opciones de respuesta con los que se evaluaron tres áreas de funcionamiento social (FP), como estrés de vida, afrontamiento y apoyo social. En la subescala del estrés de vida se consideran tres aspectos: a) el número de eventos estresantes durante el año anterior, b) el estrés percibido y c) el estrés global. Esta subescala cuenta con 18 reactivos con respuesta dicotómica (sí/no). Si la respuesta afirma que ha ocurrido el evento, se debe indicar el grado de tensión percibido en una escala de cinco puntos. La subescala mostró una consistencia interna de Cronbach de 0.73. El afrontamiento se midió con seis preguntas que identifican afrontamiento a problemas y afrontamiento a emociones en formato tipo Likert y presentó un alpha de Cronbach de 0.75 . El apoyo social se

\section{Cuadro 1}

\begin{tabular}{|c|c|c|}
\hline & $\chi=43.35$ & $\mathrm{DT}=13.1$ \\
\hline & $n(276)$ & $\%$ \\
\hline \multicolumn{3}{|l|}{ Características descriptivas } \\
\hline $\begin{array}{l}\text { Edad } \\
18-29 \\
30-49 \\
50-65\end{array}$ & $\begin{array}{r}51 \\
119 \\
105\end{array}$ & $\begin{array}{l}18.5 \\
43.1 \\
38.0\end{array}$ \\
\hline $\begin{array}{l}\text { Estado civil } \\
\text { Casado } \\
\text { Unión libre } \\
\text { Viudo } \\
\text { Soltero } \\
\text { Separado }\end{array}$ & $\begin{array}{r}179 \\
24 \\
7 \\
52 \\
12\end{array}$ & $\begin{array}{r}64.0 \\
8.7 \\
2.5 \\
18.8 \\
4.3\end{array}$ \\
\hline $\begin{array}{l}\text { Área de residencia } \\
\text { Urbana } \\
\text { Rural }\end{array}$ & $\begin{array}{r}257 \\
19\end{array}$ & $\begin{array}{r}93.0 \\
7.0\end{array}$ \\
\hline Características sociodemogr & & \\
\hline $\begin{array}{l}\text { Grado escolaridad } \\
\text { Primaria } \\
\text { Secundaria } \\
\text { Preparatoria } \\
\text { Carrera técnica } \\
\text { Profesional }\end{array}$ & $\begin{array}{r}61 \\
41 \\
34 \\
25 \\
112\end{array}$ & $\begin{array}{r}23.0 \\
14.0 \\
12.0 \\
9.0 \\
40.0\end{array}$ \\
\hline $\begin{array}{l}\text { Ingreso mensual } \\
\text { Menos de mil } \\
\text { Entre mil y tres mil } \\
\text { Entre tres mil y cinco mil } \\
\text { Entre cinco mil y diez mil } \\
\text { Diez mil o más }\end{array}$ & $\begin{array}{l}26 \\
72 \\
91 \\
60 \\
27\end{array}$ & $\begin{array}{r}9.4 \\
26.0 \\
33.0 \\
21.0 \\
9.8\end{array}$ \\
\hline $\begin{array}{l}\text { Con quién vive } \\
\text { Esposa e hijos } \\
\text { En pareja sin hijos } \\
\text { Padres e hijos } \\
\text { Padres } \\
\text { Solo } \\
\text { Otros }\end{array}$ & $\begin{array}{r}143 \\
47 \\
19 \\
22 \\
20 \\
35\end{array}$ & $\begin{array}{r}51.0 \\
16.0 \\
7.0 \\
8.0 \\
7.0 \\
22.0\end{array}$ \\
\hline
\end{tabular}


midió con 13 preguntas sobre apoyo emocional, tangible e informacional, y el coeficiente de alfa de Cronbach estuvo arriba de 0.80. Para la operacionalización del intercambio negativo definido como encuentros desagradables que se caracterizan por conflictos, demandas excesivas y/o críticas, se empleó la subescala de cuatro reactivos, mostrando un alpha de 0.77 .

d) Escala de funcionamiento en salud (EFS). ${ }^{36}$ Se utilizó, de éstos sólo la subescala que mide los hábitos positivos $\mathrm{y}$ negativos en salud y revela patrones conductuales relacionados con el uso de sustancias, hábitos alimenticios y ejercicio, lo que permite identificar los excesos o carencias en comportamiento saludable. Esta subescala consta de 24 reactivos con formato tipo Likert: 14 para hábitos positivos $(\alpha=0.64)$ y 10 para hábitos negativos $(\alpha=0.60)$.

e) Cuestionario Breve para Identificar Motivo de Consulta, con cuatro preguntas de respuesta abierta.

Además, se identificó con tres preguntas al final del cuestionario si el entrevistado acudía a atención psicológica o había recibido diagnóstico de ansiedad o depresión.

\section{Procedimiento}

Se realizó un pilotaje en una muestra de 45 participantes para verificar la confiabilidad de los instrumentos utilizados, así como la comprensión de los reactivos. Se procedió a la aplicación a la muestra total y para ello las entrevistas fueron realizadas por el propio investigador y un colaborador (ambos licenciados en psicología). Los participantes se seleccionaron en las salas de espera en forma aleatoria sistemática antes de entrar a consulta con su médico, invitándoseles a colaborar de manera voluntaria. A quien aceptó participar, se le entregó el consentimiento informado y posteriormente el investigador daba lectura a las instrucciones y a la aplicación del instrumento completo a modo de entrevista estructurada, con una duración entre 40-50 minutos.

\section{RESULTADOS}

\section{Frecuencia de síntomas de ansiedad, síntomas de depresión, factores psicosociales y motivo de consulta}

De acuerdo con los resultados, se resume que de los hombres que solicitaron atención de salud en los centros de primer nivel en Ciudad Victoria, 57\% ( $n=157)$ presentó síntomas de ansiedad $(\bar{X}=4.04 ; D T=2.97)$ y un 51\% ( $n=141)$, síntomas de depresión $(\bar{X}=2.21 ; \mathrm{DT}=2.52)$ (cuadro 2). Además, $42 \%(n=115)$ de los entrevistados presentaron sintomas mixtos de ansiedad y depresión.

En cuanto a los factores psicosociales, en estrés de vida se observa que los hombres encuestados comunican haber vivido el último año tres acontecimientos de vida estresantes como promedio $(\bar{X}=3.39$; $D T=2.28)$, siendo estos: problemas financieros $(\bar{X}=1.49 ; \mathrm{DT}=1.86)$, enfermedad grave de un familiar ( $\bar{X}=1.43$; DT=1.86) y muerte de un familiar o amigo $(\bar{X}=1.23$; DT=1.69). En cuanto a los recursos de afrontamiento, los recursos utilizados con mayor frecuencia ante eventos estresantes son: tratar de solucionar los problemas $(\bar{X}=4.18$; DT $=.77)$, tratar de sentirse mejor $(\bar{X}=4.07$; $\mathrm{DT}=.84)$, tratar de conocer mejor la situación $(\overline{\mathrm{X}}=3.77$; $\mathrm{DT}=$ $.89)$, procurar controlar la situación $(\bar{X}=3.71$; $\mathrm{DT}=.91)$, mantener la calma $(\overline{\mathrm{X}}=3.36$; $\mathrm{DT}=1.02)$ y aceptar los sentimientos $(\bar{X}=3.26 ; \mathrm{DT}=1.06)$.

Referente al apoyo social (emocional, tangible e informacional) percibido en los momentos estresantes, comunican recibir apoyo social por arriba de la media, identificando el apoyo emocional como el más recibido $(\bar{X}=11.46$; DT=2.84), expresado en muestras de afecto, estima por esposa, hijos, familiares y amigos. En contraste, refieren poca frecuencia de intercambio negativo $(\bar{X}=7.98$; $D T=3.46)$ en sus interacciones sociales.

En cuanto a los hábitos en salud, refieren practicar en promedio tanto conductas positivas $(\bar{X}=41.21$; $D T=7.19)$ que contribuyen a mantener su salud, como conductas negativas $(\bar{X}=20.95 ; \mathrm{DT}=5.52)$ que comprometen su estado de salud o que lo empeoran si es que viven con una enfermedad crónica.

Referente al motivo de consulta, los hombres refieren acudir con el médico de atención primaria en similar porcentaje tanto por enfermedad crónica (hipertensión, diabetes, epilepsia, etc.) $48 \%$ ( $n=132)$, como por otros padecimientos no crónicos (infecciones, algias y otros) 52\% ( $n=144)$. En cuanto a la frecuencia, $50.6 \%(n=140)$ solicita mensualmente este tipo de atención.

Cuadro 2. Factores psicosociales, estrés- recurso de afrontamiento y apoyo social

\begin{tabular}{lrrrr}
\hline & & & \multicolumn{2}{c}{ Puntuación } \\
\cline { 4 - 6 } & $\bar{X}$ & DT & Mínima & Máxima \\
\hline Eventos estresantes & 3.39 & 2.28 & 0 & 17 \\
Estrés percibido de los eventos & 10.34 & 8.22 & 17 & 85 \\
$\quad$ Estrés global & 2.89 & .89 & 1 & 5 \\
Eventos percibidos con más estrés & & & & \\
$\quad$ Problemas financieros & 1.49 & 1.86 & 1 & 5 \\
$\quad$ Enfermedad de un familiar & 1.43 & 1.86 & 1 & 5 \\
$\quad$ Muerte de un familiar o amigo & 1.23 & 1.69 & 1 & 5 \\
Recursos de afrontamiento & 22.35 & 3.62 & 6 & 30 \\
Apoyo social & & & & \\
$\quad$ Apoyo social total & 31.49 & 7.93 & 9 & 45 \\
$\quad$ Apoyo social informativo & 10.54 & 3.27 & 3 & 15 \\
$\quad$ Apoyo social emocional & 11.46 & 2.84 & 3 & 15 \\
Apoyo social tangible & 9.49 & 3.39 & 3 & 15 \\
Intercambio negativo & 7.98 & 3.46 & 4 & 20 \\
Hábitos de salud & & & & \\
$\quad$ Hábitos positivos & 41.21 & 7.19 & 0 & 70 \\
Hábitos negativos & 20.95 & 5.52 & 0 & 50 \\
\hline
\end{tabular}


Cuadro 3. Relación entre diagnóstico de síntomas de ansiedad y síntomas de depresión con factores psicosociales

\begin{tabular}{lccccccc}
\hline & $\begin{array}{c}\text { Total } \\
\text { de eventos } \\
\text { estresantes }\end{array}$ & $\begin{array}{c}\text { Estrés } \\
\text { percibido de } \\
\text { los eventos }\end{array}$ & $\begin{array}{c}\text { Recursos } \\
\text { de } \\
\text { afrontamiento }\end{array}$ & $\begin{array}{c}\text { Apoyo } \\
\text { social } \\
\text { positivo }\end{array}$ & $\begin{array}{c}\text { Intercambio } \\
\text { social } \\
\text { negativo }\end{array}$ & $\begin{array}{c}\text { Hábitos } \\
\text { positivos } \\
\text { de salud }\end{array}$ & $\begin{array}{c}\text { Hábitos } \\
\text { negativos } \\
\text { de salud }\end{array}$ \\
\hline Ansiedad & $\mathbf{3 5 4 *}$ & $\mathbf{. 4 6 6}^{* *}$ & .001 & $\mathbf{- 1 5 7}^{* *}$ & $\mathbf{. 3 2 2}^{* *}$ & $\mathbf{- . 2 3 8 * *}$ & $\mathbf{. 2 8 5}^{* *}$ \\
(Sig. bilateral) & .000 & .000 & .984 & .009 & .000 & .000 & .000 \\
Depresión & $\mathbf{3 2 4 * *}$ & $\mathbf{. 4 5 9 * *}$ & -.039 & $\mathbf{- . 2 2 0 * *}$ & $\mathbf{. 2 7 2 * *}$ & $\mathbf{- . 1 9 3 * *}$ & $\mathbf{. 1 2 7}^{*}$ \\
(Sig. bilateral) & .000 & .000 & .524 & .000 & .000 & .001 & .034 \\
$\mathbf{n}$ & 276 & 276 & 276 & 276 & 276 & 276 & 276 \\
\hline
\end{tabular}

* La correlación es significativa al nivel 0.05 (bilateral).

** La correlación es significativa al nivel 0.01 (bilateral).

${ }^{*} p<0.05,{ }^{* *} p<0.01$.

\section{Relación de síntomas de ansiedad y depresión con las variables sociodemográficas}

Se realizaron análisis de chi-cuadrada para ver la posible asociación de las variables sociodemográficas con las variables de síntomas de ansiedad y de depresión. No se encontró una asociación entre las variables estado civil, área de residencia y personas con las que se vive con las variables síntomas de ansiedad y depresión ( $\mathrm{p}>0.05)$. Sin embargo, sí se encontró una asociación entre la variable síntomas de depresión y las variables edad $\left(\mathrm{X}^{2}=11.049, \mathrm{p}=0.004\right)$ e ingreso mensual $\left(\mathrm{X}^{2}=21.815, \mathrm{p}<0.001\right)$.

\section{Relación de síntomas de ansiedad y depresión con factores psicosociales}

La relación entre los síntomas de ansiedad y depresión y los factores psicosociales se describe en el cuadro 3. Los resultados indican relaciones significativas, donde cuanta mayor sintomatología de ansiedad y depresión, mayores eventos estresantes, estrés percibido, intercambio negativo y hábitos negativos de salud. En cuanto a las relaciones negativas, se observa que, cuando hay una mayor sintomatología de ansiedad y depresión, hay un menor apoyo social positivo y menores hábitos positivos de salud.

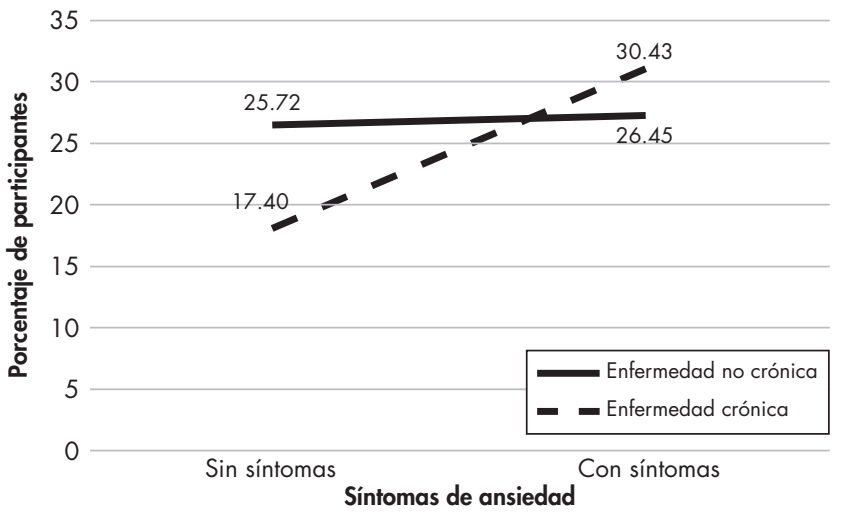

Figura 1. Relación entre presentar o no síntomas de ansiedad y el motivo de consulta.

\section{Relación entre motivo de consulta y síntomas de ansiedad y depresión}

En cuanto a la relación del motivo de consulta con los síntomas de ansiedad y depresión (figura 1), se observa una relación significativa $\left(\chi^{2}=4.703, \mathrm{gl}=1, \mathrm{p}=0.030\right)$ con los síntomas de ansiedad, donde tanto los hombres que acuden a consultar al médico por un padecimiento crónico como los que solicitan atención por una enfermedad no crónica presentan síntomas de ansiedad, siendo más elevado el porcentaje de pacientes con estos síntomas en aquellos que presentan enfermedad crónica. No se encuentra una relación significativa entre el motivo de consulta y los síntomas de depresión $\left(\chi^{2}=2.92, \mathrm{gl}=1, \mathrm{p}<0.088\right)$.

\section{Variables psicosociales predictoras de síntomas de ansiedad y depresión en varones}

El cuadro 4 muestra las variables predictoras de presentar síntomas de ansiedad, que son: el estrés percibido de los eventos (Exp [B]=1.121, IC de 95\% [1.073, 1.170], p<0.001), el intercambio negativo (Exp [B]=1.104, IC del 95\% [1.011, 1.205], $\mathrm{p}=0.028)$ y el total de hábitos negativos en salud (Exp $[\mathrm{B}]=1.067$, IC de 95\% [1.010, 1.126], $\mathrm{p}=0.020)$. Respecto al ajuste de este modelo, el cambio significativo en la desvianza muestra un ajuste global $\left(\chi^{2}=61.351, p<0.001\right)$, confirmado con el Test de Hosmer-Lemeshow $\left(\chi^{2}=10.613, \mathrm{p}=0.225\right)$, y permite hacer una estimación correcta de $70.3 \%$ de los casos. Por otro lado, el coeficiente $\mathrm{R}^{2}$ de Nagelkerke indica que $26.7 \%$ de la variabilidad en la variable dependiente se explica por el modelo de regresión logística.

Las variables predictoras de presentar síntomas de depresión son: el estrés percibido de los eventos (Exp [B]=1.099, IC de $95 \%$ [1.056, 1.144], p<0.001), el intercambio negativo (Exp [B] $=1.126$, IC de 95\% [1.036, 1.225], p<0.01) y la variable ingreso, donde un importante factor de riesgo para presentar síntomas de depresión es tener un ingreso inferior a 1000 pesos mensuales (Exp [B]=9.170, IC de 95\% [2.232, 37.676], $\mathrm{p}<0.01)$. Respecto al ajuste de este modelo, el cambio significativo en la desvianza muestra un ajuste global $\left(\chi^{2}=67.440\right.$, 
Cuadro 4. Modelo de regresión logística para las variables síntomas de ansiedad y síntomas de depresión

\begin{tabular}{lrrrrrrr}
\hline & & & & & & & $95 \%$ I.C \\
\cline { 5 - 8 } & B & S.E. & Wald & sig & Exp (B) & INF & SUP \\
\hline Ansiedad & & & & & & & \\
Estrés percibido de los eventos & .114 & .022 & 26.491 & .000 & 1.121 & 1.073 & 1.170 \\
Intercambio negativo & .099 & .045 & 4.831 & .028 & 1.104 & 1.011 & 1.205 \\
Total hábitos negativos & .065 & .028 & 5.451 & .020 & 1.067 & 1.010 & 1.126 \\
Depresión & & & & & & & \\
Estrés percibido de los eventos & .094 & .020 & 21.711 & .000 & 1.099 & 1.056 & 1.144 \\
Intercambio negativo & .119 & .043 & 7.694 & .006 & 1.126 & 1.036 & 1.223 \\
Ingreso (menos de mil mensuales) & 2.216 & .721 & 9.446 & .002 & 9.170 & 2.232 & 37.676 \\
\hline
\end{tabular}

p<0.001), confirmado con el Test de Hosmer-Lemeshow $\left(\chi^{2}=3.787, \mathrm{p}=0.876\right)$ y permite hacer una estimación correcta de $70.5 \%$ de los casos. Por otro lado, el coeficiente $\mathrm{R}^{2}$ de Nagelkerke indica que el modelo de regresión logística explica $29 \%$ de la varianza de la variable dependiente.

\section{DISCUSIÓN Y CONCLUSIÓN}

Esta investigación pretende avanzar en el conocimiento del marco social enfocándose en los sucesos vitales en relación con los síntomas de ansiedad o depresión en los hombres. Cabe aclarar que México dispone de poca información epidemiológica de los padecimientos mentales en atención primaria, y, de acuerdo con De la Fuente, ${ }^{37}$ para hacer una estimación de los trastornos mentales que afectan a la población se dispone de tres fuentes de información: las instituciones especializadas, los servicios de consulta externa de los hospitales generales y las unidades de primer nivel de atención.

Los resultados indicaron que cinco de cada 10 hombres participantes en el estudio presentaron síntomas de ansiedad y depresión. Además, cuatro de cada 10 ya está presentando síntomas mixtos (ansiedad/depresión). Utilizando una metodología análoga a la de este estudio, Enríquez et al, ${ }^{33}$ estudiaron la prevalencia de síntomas depresivos en los servicios ambulatorios de primer nivel de atención del Instituto de Salud Pública en el Estado de Guanajuato y así se identificó en los hombres mayor proporción en el grupo de depresión moderada, con $43.75 \%$, y la menor proporción fue en el grupo de la depresión grave, con $25 \%$, con lo que se concluyó que la prevalencia encontrada entre géneros era similar.

Por lo tanto, es indispensable una evaluación biopsicosocial con instrumentos de cribado a los usuarios que acudan por cualquier motivo de consulta al médico general en primer nivel, ya que los trastornos de ansiedad y del estado de ánimo son los más frecuentes en atención primaria, ${ }^{32,38}$ permitiendo un diagnóstico diferencial indispensable para la mejor opción de tratamiento.

En este estudio los hombres con síntomas de ansiedad, depresión o ambos refirieron haber experimentado más eventos estresantes el año pasado y más estrés global, resultados similares a lo reportado por estudios realizados en Estados Unidos y Canadá. ${ }^{39,40}$

Los participantes percibieron como estresores importantes: problemas financieros, enfermedad grave de un familiar y muerte de un familiar o amigo. Tomando los datos sociodemográficos de la muestra estudiada, se observa bajo grado de escolaridad $(60 \%$ sin estudios de licenciatura) y un bajo ingreso mensual (68\% percibe menos de cinco mil pesos). Aunado a esto, las condiciones económicas del país ponen en riesgo el empleo formal, lo que se refleja en la población desempleada tanto de jóvenes como adultos desplazados que no logran su inserción al mercado laboral. ${ }^{41}$ Éstas son experiencias que constituyen fuentes de malestar o incertidumbre en los hombres, con repercusión en su identidad de género de proveedores económicos. Al respecto, como se pudo observar en este estudio, tanto la edad como el ingreso mensual se relacionaron con la presencia de síntomas de depresión. Estos datos coinciden con otros estudios, ${ }^{42,43}$ donde identifican como factores de riesgo para ansiedad y depresión el desempleo, el nivel de estudios o tener un empleo menos calificado. Además, se ha demostrado consistentemente en estudios epidemiológicos que los trastornos psiquiátricos impactan en el nivel socioeconómico de quienes los padecen. ${ }^{44,45}$

Los resultados en cuanto al apoyo social fortalecen la evidencia empírica de la relación que el apoyo social tiene con la promoción de la salud mental y física en forma directa, aumentando la resistencia al estrés crónico y a los eventos importantes de la vida. Los resultados indican que los hombres refieren un deterioro en la percepción del apoyo social positivo cuando hay mayores síntomas de ansiedad y depresión. Esta asociación encontrada en otras investigaciones ha mostrado menores tasas de recuperación de la depresión en los pacientes que perciben un deficiente apoyo social, particularmente en varones de mediana edad. ${ }^{46}$ En relación con la salud física, el déficit de apoyo social se asocia con la salud cardiovascular, incluyendo infarto al miocardio y los accidentes cerebrovasculares, ${ }^{47,48}$ y a su vez puede dificultar la adherencia a la medicación. ${ }^{49}$ 
En cuanto al intercambio social negativo, los resultados indican que los hombres que reportaron síntomas de ansiedad o depresión vivieron con más rechazo críticas e insensibilidad de quienes los rodean; además, percibieron más estrés de vida y más estrés percibido de los eventos.

En relación con el motivo de consulta y los síntomas de ansiedad y depresión, tanto los hombres que acuden a consultar al médico por un padecimiento crónico como los que solicitan atención por una enfermedad no crónica presentan síntomas de ansiedad, lo que aumenta estos síntomas en los hombres que presentan una enfermedad crónica. Sin embargo, esta relación no se encuentra en los síntomas de depresión.

Los resultados indicaron que los factores psicosociales predictores de riesgo de presentar síntomas de ansiedad y depresión en los hombres, son aquéllos asociados con un mayor estrés percibido de los eventos y mayor intercambio negativo, y sólo en los síntomas de ansiedad otro factor de riesgo es la práctica de hábitos negativos de salud. Mientras, en los síntomas de depresión un factor psicosocial de riesgo es tener un ingreso mensual menor a 1000 pesos.

Se resalta que existe una necesidad apremiante en investigación tanto en prevalencia como en tratamiento de la salud mental masculina, ya que existe una relativa escasez de estudios centrados en la experiencia de los hombres, lo que plantea varios problemas de salud pública. ${ }^{50,51}$ Primero, las estimaciones basadas en la población indican que hay un número significativo de hombres que viven con depresión. Por otro lado, quienes investigan la salud mental masculina demuestran consistentemente que los hombres acuden con menor frecuencia que las mujeres a utilizar los servicios de salud mental,52 además refieren que la depresión mayor puede estar enmascarada. ${ }^{53}$ Por tanto, la patología mental no atendida en los varones se vuelve un factor de riesgo por sus consecuencias negativas no sólo para los propios hombres, sino para las mujeres, los niños y la comunidad. ${ }^{54}$

Dentro de las limitaciones de este estudio que hay que tomar en cuenta en futuras investigaciones se encuentra que el instrumento utilizado para medir depresión y ansiedad es de cribado de síntomas y no de diagnóstico del trastorno. Además, son pocos los usuarios masculinos que desean participar y los espacios abiertos, como las salas de espera, puede haber influido en la veracidad de sus respuestas.

\section{Financiamento}

Ninguno.

\section{Conflicto de intereses}

Los autores declararon no tener conflicto de intereses.

\section{Agradecimientos}

Los autores agradecen la colaboración de los centros de salud: Hospital General Dr. Norberto Treviño Zapata, Clínica Hospital del Instituto de Seguridad y Servicios Sociales de los Trabajadores del Es- tado (ISSSTE) y los 14 Centros de Salud Urbanos pertenecientes a la Jurisdicción Sanitaria N 1 en Ciudad Victoria, Tamaulipas (México).

\section{REFERENCIAS}

1. Secretaría de Salud. Rendición de cuentas en salud 2009. México, DF: Secretaría de Salud de México; 2010[acceso el 20 de septiembre de 2012]. Disponible en: http://www.dged.salud.gob.mx.

2. Secretaría de Salud. Perfil epidemiológico de la salud mental en México. México, DF: Secretaría de Salud de México; 2012 [acceso el 8 de enero de 2013]. Disponible en: http://www.epidemiologia.salud.gob.mx.

3. Medina-Mora ME, Borghes G, Lara-Muñoz C, Benjet C et al. Prevalencia de trastornos mentales y uso de servicios: Resultados de la Encuesta Nacional de Epidemiología Psiquiátrica en México. Salud Mental 2003;26(4):1-16.

4. Cassano P, Fava M. Depresion and public health: an overview. J Psychosom Res 2002;53(4):849-857.

5. Organización Mundial de la Salud. Informe sobre la salud en el mundo 2001. Salud mental. Nuevos conocimientos. Nuevas esperanzas. OMS. Ginebra: 2001 [acceso el 23 de octubre de 2012]. Disponible en: http://www.who.int/whr/2001/en/whr01_djmessage_es.pdf.

6. Egede LE, Zheng D, Simpson $\mathrm{K}$. Comorbid depression is associated with increased health care use and expenditures in individuals with diabetes. Diabetes Care 2002;25(3):464-470.

7. Cuervo A. Pautas de crianza y desarrollo socioafectivo en la infancia. Divers: Perspect Psicol 2010;6(1):111-121.

8. Sánchez MJ. Efectos traumáticos de la ausencia o disfunción paterna en un grupo de hombres adictos en tratamiento. En: Libro de ponencias: XXIV Conferencia Mundial de Comunidades Terapéuticas. Lima; Instituto del Mundo Libre; 2009; p.36-38.

9. Davies W, Isles A, Wilkinson L. Imprinted genes and mental dysfunction. Ann Med 2001;33:428-436.

10. Rin JL, Zinder M. Sexual dimorphism in mammalian gene expression. Trend Genetics 2005;21:298-305.

11. Karasu TB, Docherty P, Gelenberg A, Kupfer J et al. Practice guideline for major depressive disorder in adults. American J Psychiatry 1993;150(1):1-26.

12. Kendler K, Gatz M, Gardner C, Pedersen N. Personality and major depression: a Swedish longitudinal, population-based twin study. Arch Gen Psychiatry 2006;63(10):1113-1120.

13. Álvaro J, Garrido A, Schweiger I. Causas sociales de la depresión: una revisión crítica del modelo atributivo de la depresión. Revista Internacional Sociología 2010;68(2):333-348.

14. Kendler K, Gardner Ch, Prescott C. Toward a Comprehensive developmental model for major depression in men. Am J Psychiatry 2006;163(1):115-124.

15. Kendler K, Gardner Ch, Prescott C. Toward a comprehensive developmental model for major depression in women. Am J Psychiatry 2002;159(7):1133-1145.

16. Hamalainen J, Kaprio J, Isometsa E, Heikkinen $M$ et al. Cigarette smoking, alcohol intoxication and major depressive episode in a representative population sample. J Epidemiol Community Health 2001;55(8):573- 576.

17. Montero I, Aparicio D, Gómez-Beneyto M, Moreno-Kustner B et al. Género y salud mental en un mundo cambiante. Gaceta Sanitaria 2004;18(1):175-181.

18. Hernández H, Flores R. El suicidio en México. Papeles Población CIEAP/UAEM 2011;68:69-101.

19. Borges G, Orozco R, Benjet C, Medina-Mora ME. Suicidio y conducta suicida en México: retrospectiva y situación actual. Salud Pública Mex 2010;52(4):292-304.

20. Cochran SV, Rabinowitz FE. Gender-sensitive recommendations for assessment and treatment of depression in men. Professional Psychology: Research Practice 2003;34(2):132-140. 
21. Milani R, Parrott A, Turner J, Fox H. Gander difference in self-reported anxiety, depression, among ecstasy - MDMA polidrug users, alcohol, tobacco users, and nondrug users. Adictive Behaviors 2004;29:965-971.

22. Medina-Mora ME, Rojas E, Borges G, Vázquez-Pérez et al. Comorbidity: Depression and substance abuse. En: Aguilar-Gaxiola S, Gullotta T (eds.). Depression in Latinos. Assessment, treatment and prevention. New York: Springer, 2008.

23. NIMH [sede web]. Bethesda, MD: Instituto Nacional de Salud Mental de Estados Unidos; 2012 [acceso 19 de diciembre de 2012]. Los hombres y la depresión. Disponible en: http://www.nimh.nih.gov/health/publications/espanol/los-hombres-y-la-depresi-n/index.shtml.

24. Schafiano F, Di Furia L, Forza, G, Minicuci N et al. MDMA (ecstasy) consumption in the context of polydrug abuse: a report on 150 patient. Drug Alcohol Depend 1998;52(1):85-90.

25. Cochran SV. Evidence-based assessment with men. J Clinical Psychology 2005;61(6):649-660.

26. Iwamoto DK, Gordon DM, Oliveros A, Pérez-Cabaello MA et al. The role of masculine norms and support on mental health in incarcerated men. Psychology Men Masculinity 2012;13(3):283-293.

27. Fleiz C, Sugiyama E, Medina-Mora ME, Ramos L. Los malestares masculinos: narraciones de un grupo de varones adultos de la ciudad de México. Salud Mental 2008;31(5):381-390.

28. Agudelo D, Buela-Casal G, Spielberg Ch. Ansiedad y Depresión: El problema de la diferenciación a través de los síntomas. Salud Mental 2007;30(2):33-41.

29. Brownhil S, Wilhelm K, Barclay L. Big build: Hidden depression in men. Aust N Z J Psychiatry 2005;39(10):921-931.

30. Lara A, Medina-Mora ME, Salgado N, Acevedo M et al. Utilización de los servicios para problemas de salud mental en población femenina: Tres estudios. Salud Mental 1996;19(2):42-49.

31. Gabarrón E, Vidal J, Haro J, Boix I et al. Prevalencia y detección de los trastornos depresivos en atención primaria. Aten Primaria 2002;29:329-337.

32. Terluin B, Brouwers EPM, Van Marwijk, HWJ, Verhaak PMF et al. Detecting depressive and anxiety disorders in distressed patients in primary care; comparative diagnostic accuracy of the four-dimensional symptom questionnaire (4DSQ) and the Hospital Anxiety and Depression Scale. BMC Family Practice 2009;10:58.

33. Enríquez JF, Gonzalez FE, Manríquez PA, Rivera AE et al. Prevalencia de depresión en usuarios de los servicios ambulatorios de salud en el Estado de Guanajuato. Pensamiento Psicológico 2010;7(14):53-61.

34. Goldberg D, Bridges K, Duncan-Jones P, Grayson D. Detecting anxiety and depression in general medical settings. Br Med J 1988;297(6653):897-899.

35. Montón C, Pérez-Echevarria MJ, Campos R, Garcia-Campayo J et al. Escala de ansiedad y depresión de Goldberg: una guía de entrevista eficaz para la detección del malestar psíquico. Aten Primaria 1993;12(6):345-349.

36. Ramírez M, Cortés E, Vaquero J, Arriaga Y. Evaluación de requerimientos de salud integral en población mexicana. Psicología Ciencia Social 2003;5(2):68-75.

37. De la Fuente R, Medina-Mora ME, Caraveo J. Salud mental en México. México, DF: Instituto Mexicano de Psiquiatría/Fondo de Cultura Económica; 1997; pp.183-201.
38. González S, Fernández C, Rodríguez J, Amigo I. Prevención secundaria de la depresión en atención primaria. Psicothema 2006;18(3):471-477.

39. McLaughlin KA, Conron KJ, Koenen, KC, Gilman SE. Childhood adversity. Adult stressful life events and risk of post-yeard psychiatric disorder. A test of the stress sensitization hypothesis in a population-based sample of adults. Psichol Med 2010;40(10):1647-1658.

40. Turner R, Lloyd D. Liftime traumas and mental health: the significance accumulative adversity. J Health Soc Behav 1995:36(4):360-376.

41. Samaniego N. La crisis, el empleo y los salarios en México. ECONOMIAunam 2010;6(16):57-67.

42. Zimmerman FJ, Christakis DA, Vander A. Tinker, tailor, soldier, patient: work attributes and depression disparities among young adults. Social Science Medicine 2004;58(10):1889-1901.

43. Bello M, Puente E, Medina-Mora ME, Lozano R. Prevalencia y diagnóstico en depresión en población adulta en México. Salud pública México 2005:47(51):S4-S11.

44. Dohrenwend BP. Overview of the evidence for the importance of adverse environmental conditions in the causation of psychiatric disorders. En: Dohrenwend BO (Ed.). Adversity, stress and psychopathology. New York: Oxford University Press; 1998; pp.523-38.

45. Dohrenwend BP. The role of adversity and stress in psychopathology: some evidence and its implications for theory and research. J Health Soc Behav 2000;41:1-19.

46. George LK, Blazer DG, Hughes DC, Fowler N. Social support and the outcome of major depression. Br J Psychiatry 1989;154:478-485.

47. Lett HS, Blumenthal JA, Babyak MA, Catellier DJ et al. Dimensions of social support and depression in patients at increased psychosocial risk recovering from myocardial infarction. Int J Behav Med 2009;16(3):248-258.

48. Dafer RM, Rao M, Shareef A, Sharma, A. Poststroke depression. Top Stroke Rehabil 2008;15(1):13-21.

49. Hansen NB, Vaughan EL, Cavanaugh CE, Connell CM et al. Health-related quality of life in bereaved HIV-positive adults: relationships between HIV symptoms, grief, social support, and Axis II indication. Health Psychol 2009;28(2):249-257.

50. Ellison Ch, Zhang W, Krawe N, Marcum J. Does Negative Interaction in emotional the church Increase Depression? Longitudinal finding from the Presbyterian panel survey. Social Relig 2010;70(4):409-431.

51. Becoña E, Vázquez F, Oblitas L. Promoción de los estilos de vida saludables. Investigación en Detalle [revista en internet] 2004 [acceso el 14 de noviembre de 2012]; Número 5. Disponible en Internet <URL:http://www.alapsa.org/detalle/05/index.htm>.

52. Addis M, Cohane G. Social scientific paradigms of masculinity and their implication. J Clinical Psychology 2005;61(6):633-647.

53. Lidice J, Valdes Y, Quevedo C, Torres V et al. Depresión oculta en sujetos que laboran en condiciones complejas. Rev Cubana Med Milit 2007;36(2):1-6.

54. Calvete E. Características de salud mental de los hombres que maltratan a sus parejas. Rev Esp Sanid Penit 2008;10:49-56. 